\title{
Adalimumab: a Treatment Option for Pyoderma Gangrenosum After Failure of Systemic Standard Therapies
}

Louisa Hinterberger $\cdot$ Cornelia S. L. Müller $\cdot$ Thomas Vogt $\cdot$ Claudia Pföhler

To view enhanced content go to www.dermtherapy-open.com

Received: March 30, 2012 / Published online: May 12, 2012

(c) The Author(s) 2012. This article is published with open access at Springerlink.com

\section{ABSTRACT}

Background: Pyoderma gangrenosum (PG) belongs to a group of neutrophilic dermatoses and is often associated with systemic disorders. The authors present a patient with a recalcitrant PG that did not respond to systemic standard therapies, and discuss further treatment options with impact on quality of life.

Case Report: A 42-year-old woman with recurrent PG since 2005 was presented to the dermatology department in April 2010. Systemic standard therapies including azathioprine, corticosteroids, mycophenolate mofetil, and cyclophosphamide in

L. Hinterberger · C. S. L. Müller · T. Vogt .

C. Pföhler $(\bowtie)$

Department of Dermatology, Saarland University Hospital, Kirrbergerstrasse, 66421 Homburg/Saar, Germany

e-mail: claudia.pfoehler@uks.eu

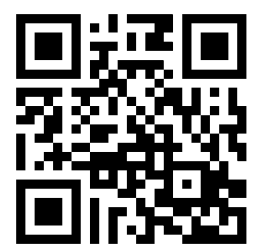

Enhanced content for this article is available on the journal web site:

www.dermtherapy-open.com combination with intensive topical treatment showed to be ineffective. Hence, in October 2010 the authors discontinued any previous systemic therapies and implemented a subcutaneously-administered therapy with adalimumab starting with $80 \mathrm{mg}$ in week 0 and $40 \mathrm{mg}$ in week 1 , followed by $40 \mathrm{mg}$ every second week. Under this therapy the ulceration got steadily smaller and healed nearly completely within 64 weeks. However, treatment with adalimumab is still ongoing, with the intent of consolidation and treatment success, but application intervals have been extended to 4-week intervals. Therapy with adalimumab was well tolerated without any side effects and led to an increased quality of life measured with the Dermatology Life Quality Index.

Discussion: An immune-modulatory monotherapy with adalimumab is a viable treatment option for recalcitrant PG. Thanks to the easy administration, rare side effects, and good healing action it is a convincing therapeutic strategy.

Keywords: Adalimumab; Pyoderma gangrenosum; Systemic therapy 


\section{INTRODUCTION}

Pyoderma gangrenosum (PG) is an inflammatory disease of uncertain etiology that belongs to a group of neutrophilic dermatoses. PG usually begins with pustules, red papules, plaques, or nodules growing rapidly to ulcerations with undermined purple-colored borders [1]. There is no typical constellation in laboratory findings nor are there specific features in histopathology. The pathergy phenomenon is often positive [1]. Extracutaneous manifestation involving mucosal structures of the upper airways, the eyes, or the genital area have been observed [2]. PG is often associated with systemic disorders such as Crohn's disease, colitis ulcerosa, rheumatoid arthritis or rheumatoid arthritislike processes, and paraproteinemia. After healing, PG lesions leave typical scars with a knitted look.

The first therapeutic step is the use of local and systemic corticosteroids. In extensive cases, steroid-sparing agents such as ciclosporine, azathioprine, dapsone, methotrexate, mycophenolate mofetil, tacrolimus, intravenously applied immunoglobulines, colchicine, or 5-aminosalicylate, can be used with unpredictable and often poor outcomes [3].

\section{CASE REPORT}

A 42-year-old woman with recalcitrant PG was first presented in the dermatology department in April 2010. Her first PG lesion was in 2005 on the lower leg, followed by an ulcer on the hypogastric region also in 2005, and in 2008 on the lower leg again. Systemic treatment with steroids (starting with a daily dosage of $1 \mathrm{mg}$ methylprednisolone/kg between 2005 and 2008) and azathioprine (100 mg daily between 2005 and 2009) had been performed in the past and resolved the lesions. A short-term treatment with ciclosporine had to be discontinued because of development of severe hypertension. Azathioprine treatment was stopped in 2009 after a period of 18 months, as the patient did not develop any new lesions. Evaluation for associated systemic diseases including gastroduodenoscopy, coloscopy, and rheumatological investigation was performed repeatedly and showed to be inconspicuous. Laboratory screening for anti-nuclear antibodies, rheumatoid factors, and anti-cyclic citrullinated peptide (CCP) antibodies was negative.

The current PG lesion on the right lower leg appeared in February 2010. At the first visit in our department the ulceration measured $15 \times 20 \mathrm{~cm}$ and the borders were purple and undermined. The patient suffered from excessive pain. Azathioprine and corticosteroid treatment during the previous 2 weeks had been unsuccessful. The authors first increased the corticosteroids (methylprednisolone in a daily dosage of $1.5 \mathrm{mg} / \mathrm{kg}$ ) in combination with azathioprine (150 mg daily) and began a systemic analgetic therapy with oxycodone. The local therapy consisted of $0.1 \%$ betamethasone ointment. At first, the lesions responded, but by lowering the dosage of methylprednisolone they got worse. Hence, the dose of systemic steroids was increased again (2 mg methylprednisolone/kg), azathioprine was kept at a daily dosage of $150 \mathrm{mg}$ per day, and mycophenolate mofetil was initiated at a daily dosage of 2,000 mg. After an initial response the ulceration became bigger $(16 \times 40 \mathrm{~cm})$, deeper, and more painful again and new small ulcerations appeared in the hypogastric region. Then, a cyclophosphamidedexamethasone pulse therapy was initiated. After the first pulse the ulcerations in the hypogastric area resolved, but after the second cycle of this therapy the patient noticed nausea, 
dizziness, and shortness of breath. Therefore, this treatment was discontinued.

After excluding any contraindications the authors implemented a subcutaneouslyadministered therapy with adalimumab starting with $80 \mathrm{mg}$ in week 0 and $40 \mathrm{mg}$ in week 1, followed by $40 \mathrm{mg}$ every second week in October 2010. The patient gave written informed consent to the off-label use of adalimumab. The ulceration measured $15 \times 30 \mathrm{~cm}$ when starting this treatment (Fig. 1a). Under this therapy, the ulceration got steadily smaller and healed nearly completely within 64 weeks (Fig. 1b). Systemic therapy with oxycodone could be lowered after 41 weeks of treatment and finally stopped in January 2012. However, treatment with adalimumab was resumed, with the intent of consolidation and treatment success, but application intervals were extended to 4-week intervals. Therapy with adalimumab was welltolerated without any side effects and led to an increased quality of life, measured with the Dermatology Life Quality Index (DLQI) (Fig. 2). To the author's knowledge this is the first case report that shows an objective improvement in quality of life by using the DLQI score.

\section{DISCUSSION}

New therapeutic strategies take into account that patients with inflammatory bowel disease, which is frequently associated with PG, present increased levels of tumor necrosis factor- $\alpha$ (TNF- $\alpha$ ). Furthermore, PG itself seems to be associated with high serum levels of TNF- $\alpha$, resulting from an abnormal T-cell response in PG patients. Hence, several authors presumed that inhibition of TNF- $\alpha$ could effectively treat PG [4-7]. In recent years, a growing rate of case reports about successful treatment of PG by the use of TNF- $\alpha$-inhibitors such as etanercept, infliximab, and adalimumab have been published.
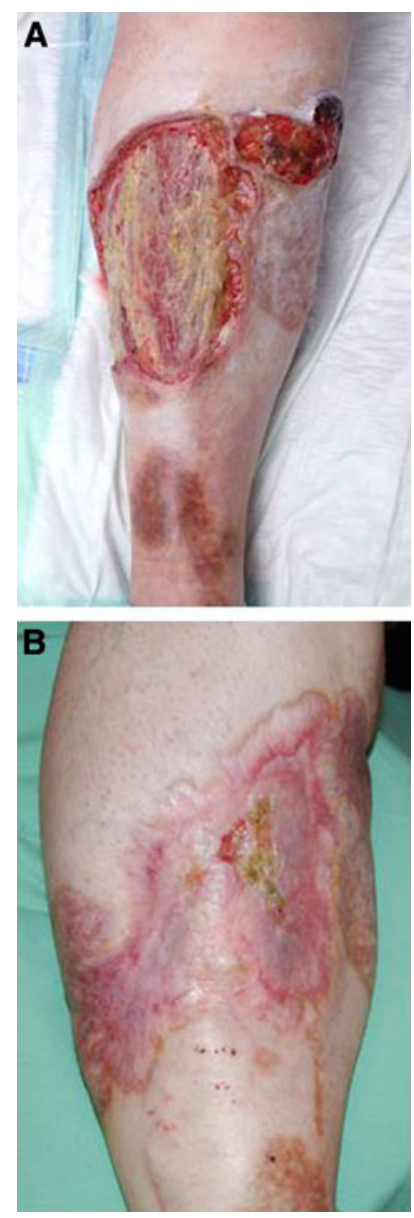

Fig. 1 Large pyoderma gangrenosum (PG) defect on the right lower leg after failure of several standard therapies and before treatment with adalimumab (a). PG almost healed after 64 weeks of treatment with adalimumab (b)

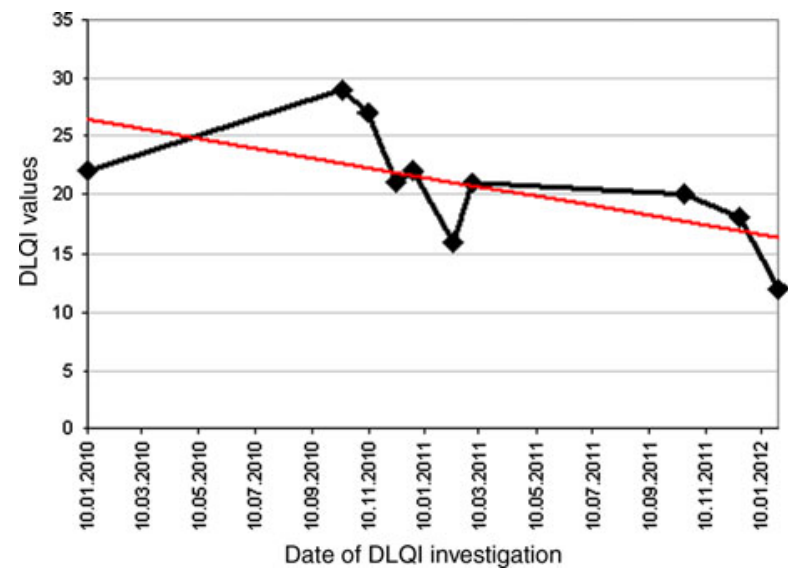

Fig. 2 Dermatology Life Quality Index (DLQI) under therapy with adalimumab. The red line shows the falling tendency of DLQI values 
For most patients, adalimumab seems to be the favored treatment option, because of the easy administration (subcutaneously and every second week only) and rare side effects. Furthermore, Hubbard et al. [6] state that the rate of antibody production against adalimumab, a fully human product, is only $12 \%$ compared to $75 \%$ in the case of infliximab, a chimeric monoclonal anti-TNF- $\alpha$ antibody. In 2007, Pomerantz et al. [7] published a case of a 61-year-old woman having a systemic reaction to infliximab at the second dose and not responding to etanercept. Under the treatment with adalimumab, initially in combination with prednisone $20 \mathrm{mg}$, the PG resolved within 5 months. Limitations of a therapy with adalimumab are infectious diseases, especially latent or active tuberculosis.

\section{CONCLUSION}

Due to several reports of successful treatment and due to the author's observations, adalimumab is a viable treatment option for recalcitrant PG. Owing to the easy administration, rare side effects and good healing action it is a convincing therapeutic strategy.

\section{ACKNOWLEDGMENTS}

Dr. Pföhler declares that there was no funding or sponsorship received in relation to this paper. Dr. Pföhler is the guarantor author for this article, and takes responsibility for the integrity of the work as a whole. There is no other person who contributed to the manuscript, other than the authors named.

Conflict of Interest. All authors declare that they have no conflict of interest.

Open Access. This article is distributed under the terms of the Creative Commons Attribution Noncommercial License which permits any noncommercial use, distribution, and reproduction in any medium, provided the original author(s) and source are credited.

\section{REFERENCES}

1. Tremezaygues L, Schmaltz R, Vogt T, Reichrath J. Management of pyoderma gangrenosum. An update on clinical features, diagnosis and therapy (in German). Hautarzt. 2010;61:345-53.

2. Sick I, Trautner B, Ruzicka T. Surgical management of pyoderma gangrenosum (in German). Hautarzt. 2011 Dec 4. (Epub ahead of print).

3. Alkhouri N, Hupertz V, Mahajan L. Adalimumab treatment for peristomal pyoderma gangrenosum associated with Crohn's disease. Inflamm Bowel Dis. 2009;15:803-6.

4. Ahronowitz I, Harp J, Shinkai K. Etiology and management of pyoderma gangrenosum: a comprehensive review. Am J Clin Dermatol. 2012;13:191-211.

5. Heffernan MP, Anadkat MJ, Smith DI. Adalimumab treatment for pyoderma gangrenosum. Arch Dermatol. 2007;143:306-8.

6. Hubbard VG, Friedmann AC, Goldsmith P. Systemic pyoderma gangrenosum responding to infliximab and adalimumab. Br J Dermatol. 2005;152:1059-61.

7. Pomerantz RG, Husni ME, Mody E, Qureshi AA. Adalimumab for treatment of pyoderma gangrenosum. Br J Dermatol. 2007;157:1274-5. 